University of South Carolina

Scholar Commons

2-8-2003

\title{
Semiclassical Dynamics with Quantum Trajectories: Formulation and Comparison with the Semiclassical Initial Value Representation Propagator
}

Sophya Garashchuk

University of South Carolina--Columbia, sgarashc@chem.sc.edu

Vitaly A. Rassolov

University of South Carolina - Columbia, rassolov@chem.sc.edu

Follow this and additional works at: https://scholarcommons.sc.edu/chem_facpub

Part of the Chemistry Commons

Publication Info

Published in Journal of Chemical Physics, Volume 118, Issue 6, 2003, pages 2482-2490.

http://jcp.aip.org/

(C) 2003 by American Institute of Physics

This Article is brought to you by the Chemistry and Biochemistry, Department of at Scholar Commons. It has been accepted for inclusion in Faculty Publications by an authorized administrator of Scholar Commons. For more information, please contact digres@mailbox.sc.edu. 


\title{
Semiclassical dynamics with quantum trajectories: Formulation and comparison with the semiclassical initial value representation propagator
}

\author{
Sophya Garashchuk and Vitaly A. Rassolov \\ Department of Chemistry and Biochemistry, University of South Carolina, Columbia, South Carolina 29208
}

(Received 7 October 2002; accepted 14 November 2002)

\begin{abstract}
We present a time-dependent semiclassical method based on quantum trajectories. Quantum-mechanical effects are described via the quantum potential computed from the wave function density approximated as a linear combination of Gaussian fitting functions. The number of the fitting functions determines the accuracy of the approximate quantum potential (AQP). One Gaussian fit reproduces time-evolution of a Gaussian wave packet in a parabolic potential. The limit of the large number of fitting Gaussians and trajectories gives the full quantum-mechanical result. The method is systematically improvable from classical to fully quantum. The fitting procedure is implemented as a gradient minimization. We also compare AQP method to the widely used semiclassical propagator of Herman and Kluk by computing energy-resolved transmission probabilities for the Eckart barrier from the wave packet time-correlation functions. We find the results obtained with the Herman-Kluk propagator to be essentially equivalent to those of AQP method with a one-Gaussian density fit for several barrier widths. (C) 2003 American Institute of Physics. [DOI: 10.1063/1.1535421]
\end{abstract}

\section{INTRODUCTION}

Quantum-mechanical (QM) effects are essential in description of nuclear motion. They become especially significant when hydrogen atoms or multiple electronic states are involved, such as in solvation dynamics, in proton transfer processes in biomolecules, in photochemistry. Due to the nonlocal character of QM traditional methods of solving the time-dependent Schrödinger equation (SE) are based on spatial grids, basis sets of functions or discrete variable representation. ${ }^{1}$ The numerical efforts for these exact QM methods scale exponentially with the dimensionality of a system. Despite many recent advances in theoretical and numerical approaches and in the computer facilities, the current state-of-the-art exact full-dimensional QM calculations have been performed for a few four-atom systems. In contrast to $\mathrm{QM}$, classical mechanics is local and the classical equations of motion for particles can be solved independently. Methods of molecular dynamics, where density is represented as an ensemble of classical particles, are applicable to systems of thousands of particles such as liquids and biosystems. The exponential scaling of exact QM methods with the dimensionality and the importance of QM effects motivate development of new approaches, that combine the simplicity of classical dynamics with the rigor of quantum mechanics.

It is natural to take advantage of the mass difference of electrons and nuclei, the Born-Oppenheimer separation, when describing molecules. First of all, it is reflected in the concept of nuclei moving on a potential electronic surface (or several coupled surfaces). Further exploitation of this separation leads to quasiclassical and semiclassical methods based on description of nuclear dynamics in terms of classical trajectories yet incorporating some QM effects. A very successful quasiclassical trajectory method ${ }^{2}$ is an ad hoc pro- cedure, where the nonclassical effects come from quantized initial conditions for the classical trajectories, and it is shown to give accurate description of total and integral cross sections and thermal rate constants in reaction dynamics, compared to experimental results and to quantum calculations. ${ }^{3}$ The Wigner phase-space transform of the quantum Liouville equation ${ }^{4}$ gives formally exact representations of QM densities and serves as the basis for various semiclassical and mixed quantum-classical methods, where the second and/or higher order terms in $\hbar$ are neglected. ${ }^{5-8}$

Another class of semiclassical methods is based on the stationary phase approximation (the limit of $\hbar \rightarrow 0$ ) to SE. The original formulation of Van Vleck proposed in 1928, expresses a semiclassical propagator in terms of classical "root" trajectories connecting points in coordinate space within a given time $t$, whose contributions depend on their stability and classical phase. This formulation with corrections of Gutzwiller and Maslov, ${ }^{9,10}$ with its multiple implementation problems of root search, coalescing trajectories and divergent amplitudes, though used extensively in theoretical analysis, never became a practical computational method.

A more successful semiclassical (SC) propagators are recast as the initial value representation (IVR) propagators (for a comprehensive overview see Ref. 11) and are based on the phase space transformation of an initial wave function and on the sampling of phase space with initial conditions for classical trajectory propagation. Trajectories are propagated independently. The classical action and stability matrix are used to determine the phase and amplitude contributions of trajectories. Better SC-IVR methods have the same $\hbar$ $\rightarrow 0$ limit as SE, i.e., the Van Vleck-Gutzwiller propagator. Currently, the most widely used SC-IVR method is the 
propagator of Herman and Kluk (HK). ${ }^{12,13}$ In the last several years the HK method was successfully applied to a wide range of problems such as double-slit experiments, photodetachment, reactive scattering, nonadiabatic and condensed phase dynamics (for instance, Refs. 14-18). One of the drawbacks is that SC-IVR methods are formulated in phase space in terms of oscillatory integrals over trajectories. To alleviate this problem various techniques of smoothing or cancellation of the integrand were suggested. ${ }^{19-22}$ Another problem is that the stability analysis is expensive and requires the second derivatives of a potential, which for a general chemical system is not available analytically. A more fundamental problem of SC methods is that it is difficult to assess and reduce the inherent semiclassical error.

A new trajectory-based alternative to traditional quantum dynamics is based on the Bohmian or quantum trajectories (QTs) that solve the hydrodynamic form of SE. ${ }^{23}$ The wave function is represented in a set of "particles" or "fluid elements" that move according to the classical equations of motion and carry along certain density. The nonlocal QM character enters this formulation by means of the quantum potential (QP), which depends on the density and its derivatives. QP governs the dynamics of the "particles" along with the classical external potential. One advantage of this description comes from the fact that the amplitude and the phase of the wave function are slowly varying functions compared to the wave function itself. Another advantage is that the solution of SE is based on trajectories, rather than grid points, and therefore the scaling bottleneck is avoided. In the last few years several practical ways of using quantum trajectories have been suggested, such as local least-square fit, adaptive and moving grids $;^{24-28}$ methodology of using QT within the Wigner representation, dissipative and nonadiabatic dynamics have been also developed. ${ }^{29-31}$ Application to several multidimensional model problems, where time evolution was accomplished with a small number of trajectories, is encouraging. However, for general problems accurate implementation of the hydrodynamic SE, which is nonlinear partial differential equation, seems impractical. A simple examination of one-dimensional systems with a barrier $^{32}$ shows, that QP becomes a rapidly varying function of large amplitude and is responsible for complicated and unstable dynamics of QTs, whenever the system undergoes drastic changes, such as a bifurcation. For example, analytical solution for the Eckart barrier ${ }^{33}$ has numerically poor convergence, when used for propagation of QTs representing a bifurcating wave packet. An illustrative example of highly complicated pattern of QTs for a surface scattering problem can be found in Ref. 34. QTs were originally proposed for the purpose of interpretation ${ }^{23}$ and were also used in the context of nonadiabatic dynamics as a theoretical ${ }^{35}$ and a practical $^{36}$ tool with QP neglected, except for the surface coupling terms.

In this paper we present the idea of using the approximate quantum potential (AQP), rather then exact QP. The central step is to fit the density in terms of Gaussian functions globally. The fitted density is used to compute QP. The accuracy of the fit controls the degree in which approximate description of QP approaches exact quantum dynamics. A
few-Gaussian fit can be performed efficiently and produces AQP, that will describe dominant quantum effects for systems characterized by the small value of $\hbar$, where semiclassical methods can improve classical results. The AQP formulation is somewhat reminiscent of the SC-IVR methods, yet there is a crucial difference: SC-IVR propagators are based on the stationary phase limit of SE, whereas our method has exact SE as its limit of high accuracy AQP.

In addition, in the present method we are able to treat the weights of trajectories as constant in a course of dynamics of a closed system. This ensures conservation of mass and is reflected in the continuity equation (3). By using constant weights we avoid errors associated with the numerical determination of the velocity gradient and time integration of the continuity equation and we are able to formulate the fitting procedure efficiently.

The formulation and the fitting procedure are outlined in Sec. II. In Sec. III we present energy-resolved transmission probabilities, determined from the wave packet timecorrelation functions for a one-dimensional Eckart barrier of several widths controlling the importance of QM effects, and compare AQP to the SC-IVR method of Herman and Kluk. Section IV concludes.

\section{DESCRIPTION OF QUANTUM POTENTIAL}

\section{A. Reformulation of the Schrödinger equation}

Equations for the Bohmian "particles" are based on the polar representation of a wave function in SE,

$$
\psi(\mathbf{x}, t)=A(\mathbf{x}, t) \exp \left(\frac{l}{\hbar} S(\mathbf{x}, t)\right)=\sqrt{\rho(\mathbf{x}, t)} \exp \left(\frac{l}{\hbar} S(\mathbf{x}, t)\right),
$$

where amplitude $A(\mathbf{x})$ and phase $S(\mathbf{x})$ are real functions. After this substitution, transformation into the Lagrangian frame of reference and identification of $\mathbf{p}=m \mathbf{v}=\nabla S(\mathbf{x}, t)$, $\mathrm{SE}$ becomes equivalent to a system of equations

$$
\begin{aligned}
& \frac{d S(\mathbf{x}, t)}{d t}=\frac{m \mathbf{v}^{2}}{2}-V-U, \\
& \frac{d \rho(\mathbf{x}, t)}{d t}=-\nabla \cdot \mathbf{v} \rho(\mathbf{x}, t),
\end{aligned}
$$

where $U$ is the nonlocal "quantum" potential

$$
U=-\frac{\hbar^{2}}{2 m} \frac{\nabla^{2} A(\mathbf{x}, t)}{A(\mathbf{x}, t)} .
$$

Equation (2) is the Hamilton-Jacobi equation of a particle of mass $m$ moving under the influence of the classical potential $V$ and the quantum potential $U$ ( $\hbar$ is set to one in expressions below). Position and momentum of the particle, $\mathbf{x}$ $=\mathbf{x}(t)$ and $\mathbf{p}=\mathbf{p}(t)$, define a quantum trajectory and can be found from Hamilton's equations of motion. Equation (1) implies that $\rho(\mathbf{x}(t), t)$ remains a single valued function at all times, i.e., the quantum trajectories do not cross. After initial discretization of the density in a set of particles, a certain amount of density within a volume element $\delta \Omega(t)$ 
$=\delta x^{(1)}(t) \delta x^{(2)}(t) \delta x^{(3)}(t) \cdots$ is associated with each trajectory and this quantity, its weight $w$, is conserved as shown in the Appendix,

$$
\rho\left(\mathbf{x}_{i}, t\right) \delta \Omega_{i}(t)=w_{i} .
$$

Conservation of the total density or the wave function normalization is

$$
\sum_{i} \rho\left(\mathbf{x}_{i}, t\right) \delta \Omega_{i}(t)=\sum_{i} w_{i}=1 .
$$

We will explicitly use Eq. (5): the weight $w_{i}$ associated with each trajectory remains constant in a course of dynamics. We do not solve Eq. (3) to determine $\rho(\mathbf{x}, t)$, which is in contrast to the standard implementations of the hydrodynamic form of SE (e.g., Refs. 24, 25, and 27), where the density is found from the continuity equation. Our approach also makes calculation of expectation value of operators especially simple: $\langle\hat{O}\rangle=\sum_{i} w_{i} O(\mathbf{x})$ with summation going over trajectories.

\section{B. Using Gaussian functions to fit QP globally}

The effects of QP on dynamics of trajectories can be broadly described as twofold. Consider neglecting QP, which is superficially an $\hbar^{2}$ term, in Eq. (2) as it is done in the Wigner approach. For a Gaussian wave packet with a linear phase at $t=0$ in the absence of external potential, the momenta of all trajectories will remain equal for all times and QM propagation will not be reproduced. Thus, one effect of QP is to produce a spread in momenta, or to "restore" the uncertainty principle on the level of position and momentum of Bohmian trajectories. A similar situation occurs for a Gaussian wave packet in a harmonic potential, a system which is reproduced exactly by the Wigner and HK methods. The two sets of trajectories-quantum trajectories with QP included and classical trajectories with $\mathrm{QP}$ neglected $(U$ $=0)$-are shown in Fig. 1(a). If the initial wave packet is narrow compared to the ground state, the effect of spreading in momentum is large, and the two sets of trajectories are quite different. Another effect of QP is manifested when we examine trajectories for an initially broad wave packet, that are shown on Fig. 1(b). Now the effect of spreading in momentum is small. Overall, the quantum and classical trajectories look similar except at the focal points, where QP generates a force that prevents quantum trajectories from crossing. For a general system, QP and the corresponding quantum force become large and rapidly oscillatory in these "avoided crossing" regions and lead to complicated and unstable dynamics. Small errors in QP will affect subsequent dynamics and lead to the crossing of trajectories and error accumulation.

Therefore, we find QP from a global fit of the density, that will be insensitive to local errors and will be performed once for all trajectories. Gaussians are chosen as fitting functions since a single-function fit readily generates correct time evolution of a Gaussian wave packet in any parabolic potential (including time-dependent or locally quadratic potentials relevant for dynamics of nuclei), as do the most successful semiclassical methods. Gaussian basis is over-complete and, thus, suitable to describe any localized nonzero density. The
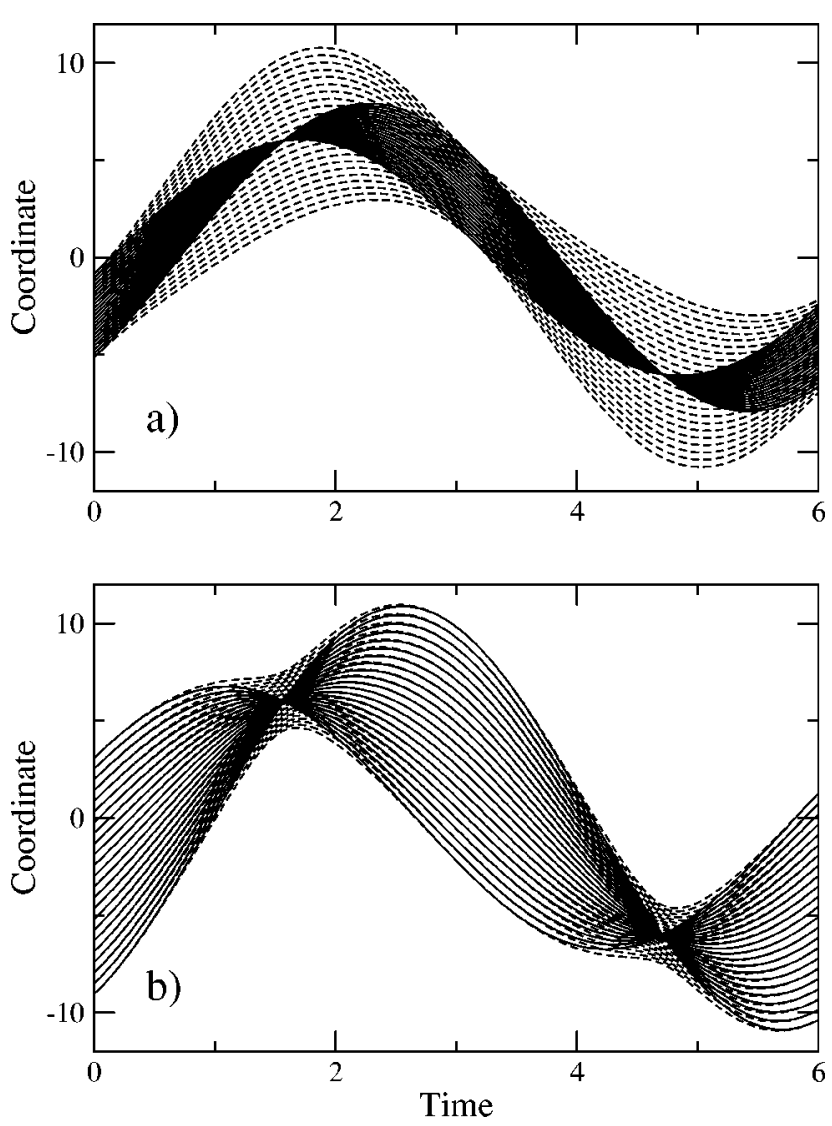

FIG. 1. Trajectories in the harmonic potential: (a) quantum (dashed line) and classical (solid line) trajectories for a narrow initial wave packet; (b) quantum (dashed line) and classical (solid line) trajectories for a wide initial wave packet.

limit of the large number of the fitting functions and trajectories is equivalent to full $\mathrm{QM}$. By selecting the maximum number of the fitting Gaussians, from which an approximate quantum potential $(\mathrm{AQP})$ is derived, we can control the accuracy and the computational effort. This strategy will give a semiclassical description with a well-defined full QM limit.

\section{Formulation of the minimization problem}

In order to determine $\mathrm{AQP}$, at each propagation time step we approximate the density $\rho(x)$ with a linear combination of Gaussian functions $g_{n}=\exp \left(-a_{n}^{2}\left(x-X_{n}\right)^{2}\right)$ as (omitting $t$ in the argument)

$$
\rho(x) \approx f(x)=\sum_{n} c_{n}^{2} g_{n} .
$$

The approximation can be of arbitrary high accuracy and is nonunique due to over-completeness of Gaussian basis. The fitting procedure is outlined for the one-dimensional case, and the multidimensional generalization is straightforward. We find a set of $g_{n}$ 's, i.e., their overall number and their parameters $s=\left\{c_{1}, X_{1}, a_{1}, c_{2}, X_{2}, a_{2}, \ldots\right\}$, from the minimization of a functional $F$,

$$
F=\int(\rho(x)-f(x))^{2} d x
$$


by solving a set of equations for the components of its gradient $\mathbf{G}$

$$
G_{k}=\frac{\partial F}{\partial s_{k}}=0
$$

or

$$
\int \rho(x) \frac{\partial f(x)}{\partial s_{k}} d x=\int \frac{\partial f(x)}{\partial s_{k}} f(x) d x .
$$

Note, that $\rho(x)$ appears on the left-hand side of the expression above in combination with $d x$. This combination can be replaced with $w_{i}$ from Eq. (5) for discrete trajectories and $\rho(x)$ will not be required explicitly. We solve Eq. (10) iteratively by minimizing the norm of the gradient, $\|\mathbf{G}\|$ $=\Sigma_{k}\left|G_{k}\right|^{2}$. The criterion is $\|\mathbf{G}\| \leqslant \epsilon$, where $\epsilon$ is a small constant (typically on the order of $10^{-11}$ ).

\section{Minimization algorithm}

\section{Convoluted density}

Discretization of the initial wave function in a set of "particles" means that $\rho(x)$ is defined only at the position of trajectories $x_{i}$. We find that the fitting can be done much more efficiently, once we define a continuous function of $\rho(x)$ - the convoluted density $\tilde{\rho}(x)$,

$$
\begin{aligned}
\tilde{\rho}(x) & =\sqrt{\frac{\beta}{\pi}} \int \exp \left(-\beta\left(x-x_{i}\right)^{2}\right) \rho\left(x_{i}\right) d x_{i} \\
& =\sum_{i} \exp \left(-\beta\left(x-x_{i}\right)^{2}\right) w_{i} .
\end{aligned}
$$

Here and below tilde symbol denotes quantities referred to a convolution. Summation goes over all trajectories. Equation (8) becomes

$$
\begin{aligned}
\widetilde{F}= & \sqrt{\frac{2 \beta}{\pi}} \sum_{i j} \exp \left(-\frac{\beta}{2}\left(x_{i}-x_{j}\right)^{2}\right) w_{i} w_{j} \\
& -2 \int \tilde{\rho}(x) \tilde{f}(x) d x+\int \tilde{f}(x)^{2} d x,
\end{aligned}
$$

where all the integrals are analytical. The equation on $\mathbf{G}$ is transformed in a similar way.

The fit to the original density $\rho(x)$ can be rigorously restored, provided the fitting Gaussians $\widetilde{g}_{n}$ are wider than the convolution Gaussian, $g_{\text {con }}(x, y)=\sqrt{\beta / \pi} \exp \left(-\beta(x-y)^{2}\right)$. The original fit $f(x)$ is obtained, if we treat $g_{n}$ as being convoluted in the same way as $\rho(x)$. The relation between the convoluted and original parameters, $a_{n}^{2}=\tilde{a}_{n}^{2} z$ and $c_{n}^{2}$ $=\widetilde{c}_{n}^{2} \sqrt{z}$ with $z=\beta /\left(\beta-\tilde{a}_{n}^{2}\right)$, imposes the restriction on the width parameters: $\tilde{a}_{n}^{2}<\beta$. In practice, for $\tilde{f}(x)$ we include $g_{n}$ 's that satisfy this condition, by assuming $\tilde{a}_{n}^{2}=\beta /(\Delta$ $\left.+\alpha_{n}^{2}\right), \Delta>1$.

Two more issues related to the suggested minimization problem are worth emphasizing. First of all, the exact QM trajectories are prevented from crossing by the precise forces exerted by QP. An AQP, in general, cannot prevent trajectories from crossing. It is, therefore, imperative that such crossings do not lead to singularities in the quantum force. The
AQP procedure using constant in time weights is formally insensitive to crossings. The overall error remains small and controllable by the parameters of the procedure, such as convolution width $\beta$ and the overall number of trajectories.

Second, equation on the gradient that we solve, the convoluted version of Eq. (10) $\widetilde{\mathbf{G}}=0$, is linear with respect to the number of trajectories. However, in our current implementation of minimization we use Eq. (12) with the double summation over trajectories to monitor the accuracy of the fit and to decide when to add new functions. This can be done less frequently than the time propagation step. In the semiclassical description of AQP with a few fitting Gaussians (SC-AQP) in Eq. (7), $\beta$ can be chosen very large. Strong cutoffs can be imposed on the double sum in Eq. (12) to make evaluation of $\widetilde{F}$ linear with the number of trajectories. Moreover, the double sum need not to be evaluated at all, once the upper limit on the number of fitting Gaussians, $N_{g}$, is reached. The accuracy of the calculation can be estimated without Eq. (12) from the normalization of $f(x)$ or from the total energy of the system, which is constant for nondissipative systems. Computation of energy is linear in the number of trajectories.

\section{The iterative procedure}

We solve the convoluted version of Eq. (10) by iterative quadratic technique with the full matrix of analytical second derivatives, derived from Newton's method to search for a zero of the gradient. ${ }^{37}$ The expansion of the gradient near its minimum is

$$
\mathbf{G} \approx \mathbf{G}_{\mathbf{0}}+A \cdot\left(\mathbf{s}-\mathbf{s}_{\mathbf{0}}\right)=\mathbf{0} .
$$

$A$ is a matrix of second derivatives, $A_{i j}=\partial^{2} F / \partial s_{i} \partial s_{j}, \mathbf{s}_{\mathbf{0}}$ is a vector of the input parameters and $\mathbf{G}_{\mathbf{0}}$ is the corresponding gradient, taken from the previous iteration or previous time step. The updated parameters are

$$
\mathbf{s}=\mathbf{s}_{\mathbf{0}}-A^{-1} \mathbf{G}_{0} .
$$

Inversion of $A$ is performed as the singular value decomposition, with a cutoff parameter imposed on the matrix eigenvalues. The difference is that all negative as well as small positive eigenvalues are replaced with the cutoff value $\left(10^{-4}\right.$ in examples below), since they indicate that $\mathbf{G}$ is not quadratic in some of the components of $\mathbf{s}_{\mathbf{0}}$ and large increments should be taken in these directions. Furthermore, in this case the new value $\widetilde{F}(\mathbf{s})$ obtained with parameters of Eq. (14) is used to scale the increment of parameters by a constant $\sigma$. Assuming a quadratic expansion over the scaling parameter $\sigma$ we have

$$
\widetilde{F}(\mathbf{s})=\widetilde{F}\left(\mathbf{s}_{0}\right)+d_{1} \sigma_{0}+d_{2} \sigma_{0}^{2},
$$

with $d_{1}=-\mathbf{G}_{0}^{T} A^{-1} \mathbf{G}_{0}$ and $\sigma_{0}=1$, from which $d_{2}=(\widetilde{F}(\mathbf{s})$ $\left.-\widetilde{F}\left(\mathbf{s}_{0}\right)\right) / \sigma_{0}^{2}-d_{1} / \sigma_{0}$. If $d_{2}>0$, the minimum of $\widetilde{F}$ is obtained for $\sigma=-d_{1} /\left(2 d_{2}\right)$ and parameters are readjusted according to $\mathbf{s}=\mathbf{s}_{\mathbf{0}}-\sigma A^{-1} \mathbf{G}$ instead of Eq. (14).

In application below, one or two iterations were sufficient to update $\mathbf{s}$ to satisfy $\|\mathbf{G}\| \leqslant \epsilon$, when the initial guess 
came from the previous time step and the number of the fitting Gaussians did not change, and 5-50 iterations otherwise.

\section{Addition of the fitting functions}

Propagation of a Gaussian wave packet starts with a single $g_{1}$ in the fit. The number of fitting Gaussians is increased each time, when $\widetilde{F}$ (or other indicator of accuracy such as total energy or normalization of the fit) exceeds a small constant, up to a maximum number of $N_{g}$. Fitting functions, whose amplitudes become small compared to the desired fitting accuracy in the course of propagation, are removed from the fit.

In order to have a good initial guess for the newly introduced fitting functions, we examine a density deviation $\delta \tilde{\rho}\left(x_{i}\right)=\tilde{\rho}\left(x_{i}\right)-\tilde{f}\left(x_{i}\right)$, defined at the position of trajectories, and generate a list of possible additional Gaussians: Their centers $X_{n}$ are located at the maxima of $\delta \tilde{\rho}\left(x_{i}\right)$, width parameters $a_{n}^{2}$ are estimated from its curvature and coefficients $c_{n}^{2}$ from its amplitude. In order to reduce the effect of convolution on the curvature estimation, the curvature is determined from the values of $\delta \tilde{\rho}\left(x_{i}\right)$ at the positions of neighboring trajectories and not from the analytical derivatives.

We have tried selecting new fitting Gaussians from this list (one or two per propagation step) according to their amplitudes $c_{n}^{2}$. In the regime of large $N_{g}$ this resulted in choosing additional fitting functions, that tended to describe features of $\tilde{\rho}$ which were too narrow for deconvolution. We have also tried using positive and negative contributions from fitting functions, $\rho \approx \sum c_{n} g_{n}$. This proved to be poorly convergent due to cancellation of contributions and could result in regions of the negative density fit. We have settled on choosing an additional function $g_{n+1}$ according to a reduction of the functional $\widetilde{F}$. A Gaussian $g_{n+1}$ which lowers $\widetilde{F}$ the most appreciably, i.e., has the largest overlap $\int-\tilde{\delta \rho}(x) g_{n+1}(x) d x$, is selected and its parameters are added to the initial guess in the optimization procedure. We choose the initial guess for its coefficient $\tilde{c}_{n+1}=\tilde{\rho}\left(X_{n+1}\right)$ to avoid introducing functions with very small compared to density amplitudes that optimize to zero. In the regime of many fitting functions, generation of initial guess for new Gaussians can affect to which particular minimum optimization converges. Fortunately, for $\mathrm{SC}-\mathrm{AQP}$ in which we are primarily interested in, optimization is quite insensitive to the initial guess.

\section{RESULTS AND DISCUSSION}

In this section, first, we illustrate the AQP method implemented with large and small number of fitting Gaussians on a one-dimensional scattering of a wave packet on the Eckart barrier. Then we formulate the time-correlation function of two wave packets within AQP framework. Finally, we compare the energy resolved transmission probabilities obtained with one, two, and four Gaussians in AQP to those obtained with the SC-IVR method of Herman and Kluk.
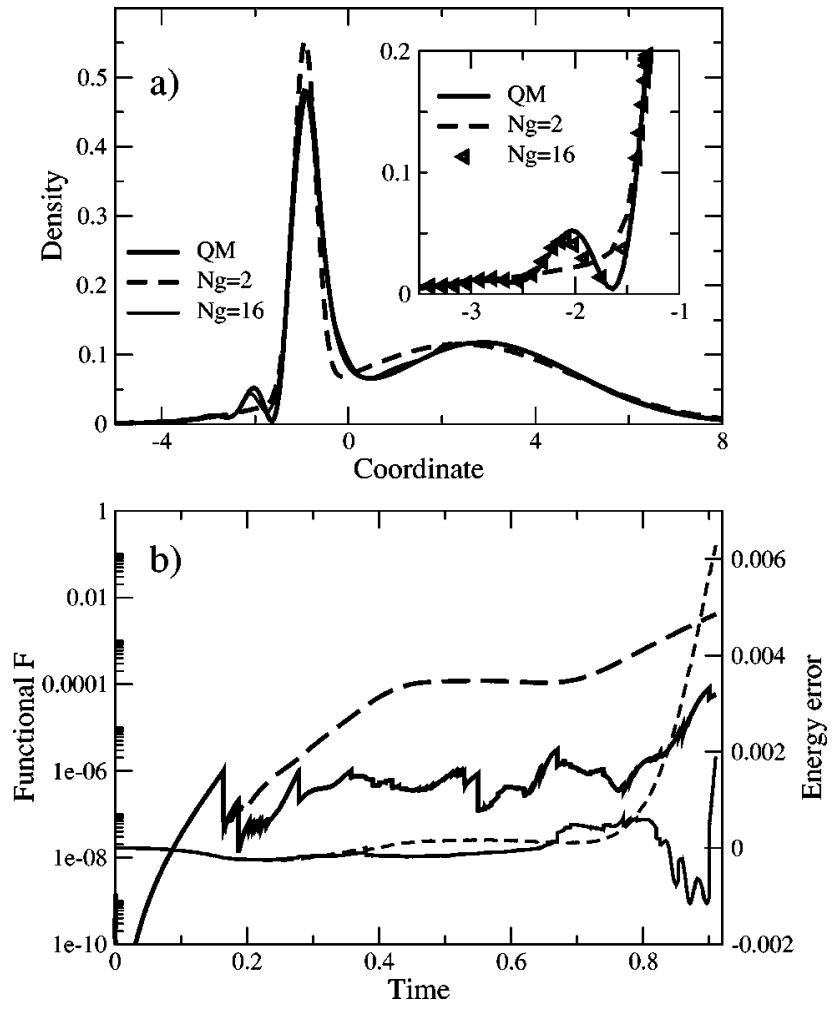

FIG. 2. (a) Density of the wave function at $t=0.9$ computed with $N_{g}=2$ and $N_{g}=16$ fitting Gaussians is shown along with the QM density. The inset shows an enlargement of the interference region. (b) The error of the density fitting, functional $\widetilde{F}$ as a function of time is shown on the logarithmic scale (left ordinate) for $N_{g}=2$ (thick dashed line) and $N_{g}=16$ (thick solid line). The relative error in the total energy (right ordinate) is shown on the linear scale for $N_{g}=2$ (thin dashed line) and $N_{g}=16$ (thin solid line).

\section{A. Density}

Let us consider a scattering of a wave packet, using the AQP method. The initial wave packet,

$$
\psi_{A}(x, 0)=\left(\frac{2 \gamma}{\pi}\right)^{1 / 4} \exp \left(-\gamma\left(x-x_{A}\right)^{2}+\imath p_{A}\left(x-x_{A}\right)\right),
$$

is located on the left of the Eckart barrier, $V(x)$ $=D \cosh ^{-2}(\lambda x)$. The parameters of the barrier, $D=16.0$ and $\lambda=1.3624$ for $m=1$, mimic hydrogen exchange reaction. Parameters are given in atomic units, with the unit of time scaled by $m_{H} / 2(\approx 918$ a.u. $)$. The wave packet parameters are $\left\{q_{A}=-3.0, p_{A}=6.0, \gamma=6\right\}$. Figure 2(a) shows the fit of the density $f(x, t)$ at time $t=0.9$ obtained with a maximum number of fitting Gaussians $N_{g}=2$ and $N_{g}=16$, as well as the accurate QM density computed with the split operator method for time-dependent SE. ${ }^{38}$ In both AQP calculations the initial number of QTs was 199, the convolution parameter was $\beta=3 \gamma$ and $\Delta=2.5$. Up to two new fitting functions per time step were allowed. Additional trajectories were introduced in the barrier region $|x|<2$, when the distance between two adjacent trajectories increased during the wavepacket bifurcation such, that their overlap dropped below $\zeta$ $=0.975$ as described in Sec. III B. The $N_{g}=2$ fit reproduces the bifurcation of the wave packet in general, but does not describe the small amplitude lobes due to interference of the 
incoming and reflected components of $\psi_{A}(x, t)$. The highaccuracy calculation with up to $N_{g}=16$ fitting Gaussians captures this pattern. The error of the fit, which is a value of the functional $\widetilde{F}$, is shown of Fig. 2(b). Over the entire timeinterval $\widetilde{F}$ is smaller than 0.005 for $N_{g}=2$ calculation and it is smaller than $10^{-5}$ for $N_{g}=16$ calculation, with the target value being set to $10^{-6}$ in both cases. The relative error of the total energy is also shown on this figure (the right vertical axes). It is under a few tenths of a percent for both calculations.

We find, that while propagation with AQP obtained with a few fitting Gaussians is fairly robust, the long-time propagation with a high-accuracy fitting becomes expensive and unstable. We use the velocity Verlet algorithm ${ }^{39}$ to propagate trajectories, which gives bounded energy error and is time reversible for classical trajectories. Due to the large space and time variations of $\mathrm{QP}$, the overall dynamics of quantum trajectories critically depends on the time step, and very small steps may be needed, even if QP were exact. For trajectory propagation using AQP with $N_{g}=16$ functions we had to reduce the time step size by a factor of 8 compared to $N_{g}=2$ calculation, for which the step size was $d t=1.25$ $\times 10^{-3}$. For high accuracy fitting the problem is that small errors of AQP are reflected in $\tilde{\rho}$ and are reproduced by the fit. In general, QP generates smooth flow of trajectories in coordinate space and pushes them apart if they tend to cross. The artificial oscillations of $\tilde{\rho}$ become smoothed out at the expense of significant reduction of the step size. Clearly, some stabilization technique is needed for the long-time highaccuracy AQP propagation. Energy conservation might be useful to ensure smoothness of the fitting parameters in time. For SC-AQP computed from one or two Gaussian density approximations, both fitting and propagation are quite stable, since with a limited number of fitting functions-one per channel-there are no functions available for fitting of smallamplitude local deviations of $\tilde{\rho}$.

\section{B. Probabilities}

In our preliminary work ${ }^{40}$ we computed the wave packet transmission probabilities (fraction of the transmitted density as a function of $p_{0}$ ) by summing over the weights of trajectories in the appropriate subspace. The required propagation time was quite short and the results did not involve phases, volume elements, etc., that made them robust. We expect that this will be true for expectation value computations. However, for quantities that involve both density and phase the situation is different. SC-AQP generates approximate dynamics of QTs, which will result in inaccurate $d \Omega_{i}$ and, consequently, in inaccurate $\rho\left(x_{i}, t\right)$ found from Eq. (5), especially if trajectories cross and $d \Omega_{i}$ is small. Therefore it is advantageous to extract information from approximate dynamics avoiding division by $d \Omega_{i}$ or bypassing $\rho\left(x_{i}, t\right)$ altogether.

As an example, we will look at the energy-resolved transmission probabilities $N(E)$, which are the central quantity of interest in reactive scattering. We compute $N(E)$ from the time-correlation function of two wave packets ${ }^{41}$

$$
N(E)=\eta(E)\left|\int_{0}^{\infty}\left\langle\psi_{B}\left|\exp \left(-{ }_{\imath} H t\right)\right| \psi_{A}\right\rangle \exp (\imath E t) d t\right|^{2},
$$

where $\left|\psi_{A}\right\rangle$ and $\left|\psi_{B}\right\rangle$ are the reactant and product wave packets, respectively. $\eta(E)$ is the appropriate energy normalization function. In terms of QTs the correlation function is

$$
\left\langle\psi_{B}(0) \mid \psi_{A}(t)\right\rangle=\sum_{i} \psi_{B}^{\star}\left(x_{i}, 0\right) \exp \left({ }_{l} S_{i}\right) \sqrt{w_{i} d \Omega_{i}} .
$$

We choose this expression over the alternatives, that can be obtained from Eq. (5), because $d \Omega_{i}$, defined as an average distance to the adjacent trajectories, enters as a small multiplicative factor. Also note, that for this problem the limit of $U=0$ will provide a poor starting point for $N(E)$. It will be always zero for $p_{A}<\sqrt{2 D}$, but not a step function which was the case for the wave-packet probabilities.

Equation (18) implies a summation of the sign-changing terms which is similar to the summation over the classical trajectories with phases and amplitudes in SC-IVR methods. In SC-IVR calculations one usually performs a Monte Carlo sampling in phase space, and the sign problem presents a major difficulty. In principle, since with $\mathrm{AQP}$ we compute the fitted density $f(x, t)$ we can replace the summation in Eq. (18) with an integral (eliminating the need for volume elements as well), if we define the action function $S_{\text {fit }}(x, t)$ for all $x$ in a way that is consistent with the density approximations. The natural way to do this is to use a local polynomial least square fit of $S\left(x_{i}\right)$ around $x_{j}$, weighted by the same convolution Gaussian $g_{\text {con }}=\sqrt{\beta / \pi} \exp \left(-\beta\left(x_{i}-x_{j}\right)^{2}\right)$ as in $\tilde{\rho}(x, t)$. A single second order fit is exact for parabolic potentials. Integration of the approximate correlation function with $f(x, t)$ and $S_{f i t}(x, t)$ can be carried out analytically. The difference between this approach and Eq. (18) is noticeable in $\mathrm{SC}-\mathrm{AQP}$ calculations, since using approximate density and phase goes beyond using AQP for time propagation, and it vanishes in the high accuracy regime.

For Eq. (18), however, with the summation going in coordinate and not in phase space as in SC-IVR methods, the problem was a mere absence of trajectories with nonzero contributions at long times, if we propagated trajectories that were equally spaced at $t=0$. This was remedied by adding trajectories in the region of bifurcation as they spread far apart, taking into account the convolution parameter $\beta$. At time $t$ a trajectory was added at $\left(x_{i}(t)+x_{i+1}(t)\right) / 2$ if the overlap, $\zeta=\exp \left(-\beta\left(x_{i+1}(t)-x_{i}(t)\right)^{2}\right)$, dropped below a certain constant. The remaining parameters, $p, S$ and $\rho$ determining $w$, for this additional trajectory were found from the quadratic fit over four nearest trajectories. If the AQP parameters as functions of time are saved, one can also define the additional trajectories at $t=0$ without fitting or interpolation steps.

\section{Comparison with the propagator of Herman and Kluk}

In order to assess the performance of SC-AQP method, we compare it to the SC-IVR propagator of Herman and 

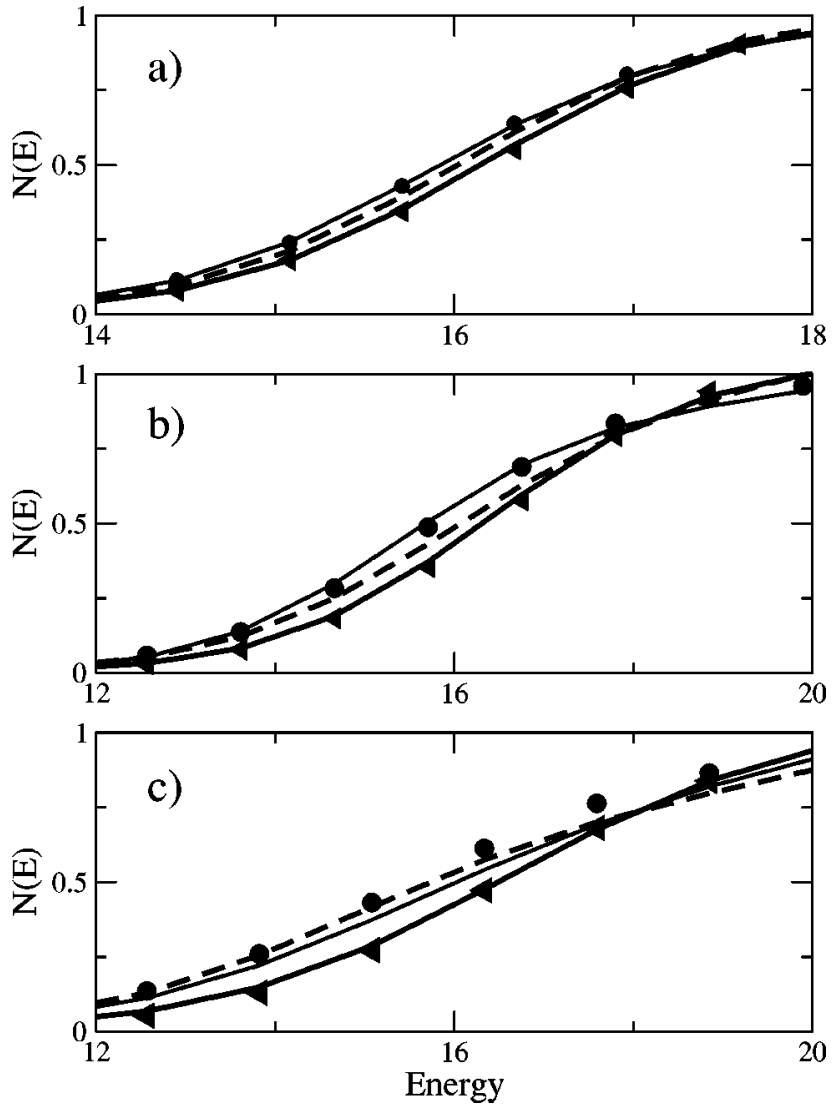

FIG. 3. Transmission probability $N(E)$, computed using $N_{g}=1$ Gaussian (thick solid), $N_{g}=2$ Gaussians (dashed line), and $N_{g}=4$ Gaussians (thin solid line). The analytical result is shown with circles and the HK probability is shown with triangles. The three panels describe three different potentials: (a) "classical" wide barrier $\lambda=0.81744$, (b) hydrogen exchange $\lambda$ $=1.36240$, and (c) "quantum" narrow barrier $\lambda=1.90736$. Note different energy range for panels (a), (b), and (c).

Kluk. The semiclassical correlation function computed with HK method has a similar structure as the formulation in terms of quantum trajectories:

$$
\begin{aligned}
\left\langle\psi_{B}(0) \mid \psi_{A}(t)\right\rangle^{H K}= & \iint d q d p\left\langle\psi_{B}(0) \mid g\left(q_{t}, p_{t}\right)\right\rangle \\
& \times \exp \left(i S_{q p t}\right)\left\langle g\left(q_{0}, p_{0}\right) \mid \psi_{A}(0)\right\rangle R_{q p t} .
\end{aligned}
$$

$g\left(q_{t}, p_{t}\right)=(\gamma / \pi)^{1 / 4} \exp \left(-\gamma / 2\left(x-q_{t}\right)^{2}+\imath p_{t}\left(x-q_{t}\right)\right) \quad$ are Gaussian functions with the width parameter $\gamma$ constant in time and $\left(q_{t}, p_{t}\right)$ are the coordinates of a trajectory with initial conditions of $\left(q_{0}, p_{0}\right) . R_{q p t}$ is a complex prefactor which is a function of the stability matrix elements of the trajectory; $S_{q p t}$ is the classical action. Integration goes over phase space. The transformation of the initial wave function into phase space gives a momentum distribution to classical trajectories, such that the uncertainty principle in the initial conditions on trajectories is fulfilled. It is this transformation at $t=0$, that introduces $\mathrm{QM}$ effects into HK propagator.

This statement is supported by the results of Fig. 3, where we show $N(E)$ computed using AQP with $N=1,2$, and 4 fitting Gaussians and the result of the HK calculation in the region, where $N(E)$ is different from the step function.
TABLE I. Cumulative deviation of AQP and HK probabilities from the analytical result, $(\Delta N)^{2}$; the largest error in the fitting procedure, $\widetilde{F}$; the initial location $x_{A}$ and total propagation time $t_{\max }$ for three values of the

\begin{tabular}{|c|c|c|c|}
\hline$\lambda$ & 0.81744 & 1.3624 & 1.90736 \\
\hline Method & \multicolumn{3}{|c|}{$(\Delta N)^{2}$} \\
\hline HK & 0.021 & 0.058 & 0.109 \\
\hline$N_{g}=1$ & 0.015 & 0.047 & 0.098 \\
\hline$N_{g}=2$ & 0.004 & 0.025 & 0.053 \\
\hline$N_{g}=4$ & 0.001 & 0.004 & 0.038 \\
\hline \multicolumn{4}{|c|}{ Maximum value of $\widetilde{F}$} \\
\hline$N_{g}=1$ & 0.140 & 0.160 & 0.165 \\
\hline$N_{g}=2$ & 0.012 & 0.014 & 0.013 \\
\hline$N_{g}=4$ & 0.009 & 0.005 & 0.008 \\
\hline \multicolumn{4}{|c|}{ Propagation parameters } \\
\hline$x_{A}$ & -5.0 & -3.0 & -2.2 \\
\hline$t_{\max }$ & 2.5 & 3.0 & 5.0 \\
\hline
\end{tabular}
barrier width $\lambda$.

We computed probabilities for three widths of the Eckart barrier: $\lambda=0.81744$ corresponds to a more "classical" case of a wide barrier, $\lambda=1.36240$ - to the hydrogen exchange reaction and $\lambda=1.90736$ - to a more "quantum" narrow barrier. The initial location of $\left|\psi_{A}\right\rangle,\left|\psi_{B}\right\rangle, x_{A}=-x_{B}$, and the total propagation time, listed in Table I, depend on the value of $\lambda$, since the wave packets have to be set up in the asymptotic region of $V$ at $t=0$. The initial momenta are $p_{A}$ $=p_{B}=6$ and $\gamma=6$ for all three cases. The propagation parameters are the same as in the density calculation of Sec. III A. The time step for $N_{g}=1,2$ was $d t=1.25 \times 10^{-2}$. It was reduced by a factor of 4,8 , and 16 for $N_{g}=4$ with $\lambda$ $=081744,1.36240,1.90736$, respectively. Deviation in probabilities for the AQP and HK probabilities from analytical $N(E)$ and accuracy of the density fit and energy conservation are summarized in Table I.

For all three values of $\lambda$ we see a remarkable agreement of the one-Gaussian AQP and HK probabilities. Their agreement with the exact result is better for more classical systems. The agreement of $N_{g}=2$ and $N_{g}=4 \mathrm{AQP}$ probabilities with the analytical QM result is improved compared to $N_{g}$ $=1 \mathrm{AQP}$ calculation. The only case when the quality of $N_{g}$ $=4$ results is not apparently superior to that of $N_{g}=2$ results is for the narrow barrier, though the cumulative deviation, $(\Delta N)^{2}=\int\left(N(E)-N(E)^{\mathrm{QM}}\right)^{2} d E$ integrated over $E$ $=[0,30]$, decreases for $N_{g}=4$ for all barriers as seen from the table. Clearly, four fitting Gaussians were not sufficiently close to the QM limit for this particular system, where QM effects are more pronounced.

The overall trend indicates that $\mathrm{SC}-\mathrm{AQP}$ calculations can efficiently and accurately describe semiclassical systems, such as in reactive scattering. Unlike the HK method quantum trajectories sample the coordinate space. The fitting of the density is performed once per time step for all trajectories. Expensive calculation of the stability matrix, which requires to solve $4 N_{d}^{2}$ equations $\left(N_{d}\right.$ is the number of dimensions) and a knowledge of the second derivative of $V$ for each classical trajectory, is not needed. From the conceptual point of view, the drawback of QP methods compared to 
SC-IVR methods is that quantum trajectories have to be propagated simultaneously. But there are major advantages: AQP method allows easy error estimation, is systematically improvable and has full QM as its high-accuracy limit.

\section{CONCLUSIONS}

We have described a new approach to approximation of quantum dynamics. Our method is based on trajectory propagation in the presence of approximate quantum potential (AQP), and it has full QM as a limit of highly accurate QP and many trajectories. By using constant in time weights for the trajectories, we avoid solving the continuity equation for the wave function density. This provides a convenient expression of expectation values and allows for an efficient fitting procedure of AQP. AQP is determined from a global fit of the density in terms of Gaussian functions. One Gaussian approximation is exact for a Gaussian wave packet in any parabolic potential, a feature that it shares with the most successful semiclassical methods.

We have also computed energy-resolved transmission probabilities for a one-dimensional scattering system using the time-correlation function of wave packets. One Gaussian approximation provides good description of tunneling and gives results identical to those obtained with the SC-IVR method of Herman and Kluk. AQP determined from the density fittings with multiple Gaussians, improves the agreement of probabilities with $\mathrm{QM}$ results. We expect that a few Gaussian (perhaps, one function per channel) SC-AQP will give an adequate description of semiclassical systems, such as in reaction dynamics, and being systematically improvable and cheap will be well suited for treatment of multidimensional semiclassical systems.

\section{ACKNOWLEDGMENTS}

The authors thank R. E. Wyatt for useful comments on the paper. This work was supported by a Grant No. EPS0296165 from NSF.

\section{APPENDIX: CONSERVATION OF DENSITY WITHIN A VOLUME ELEMENT}

In a closed system the matter is conserved and the density within a volume element remains constant in time, $\rho(\mathbf{x}, t) \delta \Omega(t)=w$, as demonstrated below. To find time dependence of $\delta \Omega(t)$, we take an infinitesimal displacement of position and velocity of the trajectory, defined by Hamilton's equations of motion, to obtain

$$
\begin{aligned}
& m \frac{d}{d t} \delta \mathbf{v}=-\nabla^{2}(V+U) \delta \mathbf{x}, \\
& \frac{d}{d t} \delta \mathbf{x}=\delta \mathbf{v} .
\end{aligned}
$$

Differentiating $\rho(\mathbf{x}, t) \delta \Omega(t)$ with respect to time $t$ and using Eq. (A2) and the continuity equation (3) along with the fact that quantum trajectories do not cross, $\delta \Omega=\delta x^{(1)} \cdot \delta x^{(2)}$ $\cdots \neq 0$, we have

$$
\begin{aligned}
\frac{d}{d t}(\rho(\mathbf{x}, t) \delta \Omega(t))= & \delta \Omega(t) \frac{d \rho(\mathbf{x}, t)}{d t}+\rho(\mathbf{x}, t) \frac{d \delta \Omega(t)}{d t} \\
= & \delta \Omega(t) \frac{d \rho(\mathbf{x}, t)}{d t}+\rho(\mathbf{x}, t)\left(\frac{d \delta x^{(1)}}{d t} \frac{1}{\delta x^{(1)}}\right. \\
& \left.+\frac{d \delta x^{(2)}}{d t} \frac{1}{\delta x^{(2)}}+\cdots\right) \delta \Omega(t) \\
= & \left(\frac{d \rho}{d t}+\rho(t) \boldsymbol{\nabla} \cdot \mathbf{v}\right) \delta \Omega(t)=0,
\end{aligned}
$$

where

$$
\boldsymbol{\nabla} \cdot \mathbf{v}=\sum_{n} \frac{\delta v^{(n)}}{\delta x^{(n)}} .
$$

This means that after discretizing the initial wave function $\psi(\mathbf{x}, 0)$ through a set of trajectories with initial positions $\left\{\mathbf{x}_{i}\right\}$, velocities $\left\{\mathbf{v}_{i}=\nabla S\left(\mathbf{x}_{i}, 0\right) / m\right\}$, densities $\left\{\rho\left(\mathbf{x}_{i}, 0\right)=A\left(\mathbf{x}_{i}, 0\right)^{2}\right\}$ and corresponding volume elements $\left\{\delta \Omega_{i}(0)\right\}$, for each trajectory the amount of density within its volume element will be conserved: $\rho\left(\mathbf{x}_{i}, t\right) \Omega_{i}(t)=\rho\left(\mathbf{x}_{i}, 0\right) \delta \Omega_{i}(0)=w_{i}$. In principle, Eqs. (A1) and (A2) give an independent way of finding the gradient of velocity and the volume element for a trajectory. In practice, their implementation might be quite cumbersome, since it requires the second derivative of the quantum potential (the fourth derivative of the density).

${ }^{1}$ J. C. Light and T. Carrington, Jr., Adv. Chem. Phys. 114, 263 (2000).

${ }^{2}$ M. Karplus, R. N. Porter, and R. D. Sharma, J. Chem. Phys. 43, 3259 (1965)

${ }^{3}$ G. C. Schatz, Theor. Chem. Acc. 103, 270 (2000).

${ }^{4}$ E. P. Wigner, Phys. Rev. 40, 749 (1932).

${ }^{5}$ O. Prezhdo and V. V. Kisil, Phys. Rev. A 56, 162 (1997).

${ }^{6}$ C. C. Martens and J. Y. Fang, J. Chem. Phys. 106, 4918 (1997).

${ }^{7}$ E. J. Heller, J. Chem. Phys. 65, 1289 (1976).

${ }^{8}$ R. C. Brown and E. J. Heller, J. Chem. Phys. 75, 186 (1981).

${ }^{9}$ J. V. Van Vleck, Proc. Natl. Acad. Sci. U.S.A. 14, 178 (1928)

${ }^{10}$ M. Gutzwiller, Chaos in Classical and Quantum Mechanics (SpringerVerlag, New York, 1990).

${ }^{11}$ W. H. Miller, J. Phys. Chem. A 105, 2942 (2001).

${ }^{12}$ M. Herman and E. Kluk, Chem. Phys. 91, 27 (1984).

${ }^{13}$ K. G. Kay, J. Chem. Phys. 100, 4377 (1994).

${ }^{14}$ R. Gelabert, X. Gimenez, M. Thoss, H. B. Wang, and W. H. Miller, J. Chem. Phys. 114, 2572 (2001).

${ }^{15}$ M. L. Brewer, J. S. Hulme, and D. E. Manolopoulos, J. Chem. Phys. 106, 4832 (1997).

${ }^{16}$ S. Garashchuk and D. J. Tannor, Chem. Phys. Lett. 262, 477 (1996).

${ }^{17}$ F. Grossmann, Phys. Rev. A 60, 1791 (1999).

${ }^{18}$ K. Thomson and N. Makri, J. Chem. Phys. 110, 1343 (1999).

${ }^{19}$ X. Sun and W. H. Miller, J. Chem. Phys. 110, 6635 (1999).

${ }^{20}$ H. B. Wang, D. E. Manolopoulos, and W. H. Miller, J. Chem. Phys. 115, 6317 (2001)

${ }^{21}$ S. Garashchuk and J. C. Light, J. Chem. Phys. 114, 1060 (2001).

${ }^{22}$ K. Thompson and N. Makri, Phys. Rev. E 59, 4729 (1999).

${ }^{23}$ D. Bohm, Phys. Rev. 85, 167 (1952).

${ }^{24}$ C. L. Lopreore and R. E. Wyatt, Phys. Rev. Lett. 82, 5190 (1999).

${ }^{25}$ R. E. Wyatt and E. R. Bittner, J. Chem. Phys. 113, 8898 (2000).

${ }^{26}$ D. Nerukh and J. H. Frederick, Chem. Phys. Lett. 332, 145 (2000).

${ }^{27}$ B. K. Dey, A. Askar, and H. Rabitz, J. Chem. Phys. 109, 8770 (1998).

${ }^{28}$ R. E. Wyatt and K. Na, Phys. Rev. E 65, 016702 (2002).

${ }^{29}$ A. Donoso and C. C. Martens, J. Chem. Phys. 115, 6309 (2002).

${ }^{30}$ E. R. Bittner, J. Chem. Phys. 115, 6309 (2002). 
${ }^{31}$ R. E. Wyatt, C. L. Lopreore, and G. Parlant, J. Chem. Phys. 114, 5113 (2001).

${ }^{32}$ P. R. Holland, The Quantum Theory of Motion (Cambridge University Press, Cambridge, 1993).

${ }^{33}$ C. Eckart, Phys. Rev. 35, 1303 (1930).

${ }^{34}$ A. S. Sanz, F. Borondo, and S. Miret-Artes, J. Phys.: Condens. Matter 14, 6109 (2002).

${ }^{35}$ J. C. Burant and J. C. Tully, J. Chem. Phys. 112, 6097 (2000).

${ }^{36}$ E. Gindensperger, C. Meier, and J. A. Beswick, J. Chem. Phys. 113, 9369 (2000).
${ }^{37}$ W. Press, B. Flannery, S. Teukolsky, and W. Vetterling, Numerical Recipes: The Art of Scientific Computing, 2nd ed. (Cambridge University Press, Cambridge, 1992).

${ }^{38}$ C. Leforestier, R. H. Bisselling, C. Cerjan et al. J. Comput. Phys. 94, 59 (1991).

${ }^{39}$ M. Tuckerman, B. Berne, and G. Martyna, J. Chem. Phys. 97, 1990 (1992).

${ }^{40}$ S. Garashchuk and V. A. Rassolov, Chem. Phys. Lett. 364, 562 (2002).

${ }^{41}$ D. J. Tannor and D. E. Weeks, J. Chem. Phys. 98, 3884 (1993). 